\title{
The Targeting Accuracy of the CyberKnife as Measured with EBT2 Film Inside a Ball- Cube-II Film Cassette and Head Phantom
}

\author{
Anthony K. Ho ${ }^{1}$, Jiji Antony ${ }^{2}$, John R. Adler ${ }^{3}$ \\ 1. Department of Radiation Oncology, Stanford University School of Medicine 2. Cyberknife Of \\ Birmingham 3. Department of Neurosurgery, Stanford University School of Medicine
}

$\square$ Corresponding author: Anthony K. Ho, tony22006@gmail.com

Disclosures can be found in Additional Information at the end of the article

\section{Abstract}

Introduction: The CyberKnife (Accuray, Inc., Sunnyvale, California, USA) is an image-guided radiosurgical system which utilizes a compact 6-MV linear accelerator mounted on a robotic arm to deliver radiosurgery. The total system error of the CyberKnife system, as measured with an anthropomorphic head phantom containing a film holder, has been previously reported to be submillimetric. In making these prior accuracy measurements, the Ball-Cube-I was used. However, a new design, the Ball-Cube-II, which uses a different kind of pre-cut films, has recently become available. In this study, we investigate the use of this device for measuring the total system accuracy of the CyberKnife.

Methods: The Ball-Cube-II film cassette is a radiosurgical quality assurance (QA) tool. Similar to the existing Ball-Cube-I design, the Ball-Cube-II holds two pieces of notched radiochromic (www.gafchromic.com) film that are placed orthogonally within the film cassette. There are holes and pins in the film Ball-Cube-II cassette and holes in the EBT2 film, allowing the film to fit tightly, thus minimizing movement of the film with respect to the film cassette. The ball cube is then placed within an anthropomorphic phantom. The method for determining the localization accuracy of the CyberKnife System is similar for both film cube designs. More specifically, the spatial coordinates for the centroid of the planned dose distribution is compared with the centroid of the dose actually delivered.

Results: Using the Ball-Cube-I with MD-55 film, and the head phantom, the total accuracy measured was $0.34+/-0.17 \mathrm{~mm}$. While using the ball-cube II with EBT2 film, the total accuracy measured was $0.45+/-0.09 \mathrm{~mm}$.

Conclusions: The ball-cube-II restricts movement of the film inside the film cube, and thereby reduces the uncertainty of film placement within the cube. This design has the potential to more precisely determine the accuracy of the CyberKnife system. The accuracy of the CyberKnife using film cube with the head phantom was measured to be between 0.34 and 0.45

Published 10/29/2009

(c) Copyright 2009

Ho et al. This is an open access article distributed under the terms of the Creative Commons Attribution License CC-BY 3.0., which permits unrestricted use, distribution, and reproduction in any medium, provided the original author and source are credited. $\mathrm{mm}$.

Categories: Medical Physics, Radiation Oncology

Keywords: cyberknife, image guidance, physics, quality assurance, radiation oncology, radiosurgery

\section{Introduction}

The CyberKnife (Accuray, Inc., Sunnyvale, California, USA) is an image-guided radiosurgery 


\section{Cureus}

system [1]. It uses a compact 6-MV linear accelerator mounted on a robotic arm to deliver radiosurgical doses with submillimeter accuracy [2]. Changes in patient position are detected by image-guidance using a pair of x-ray cameras (amorphous silicon detectors) and x-ray sources. These images are compared by computer to digital reconstructed radiographs (DRR) generated from planning computed tomography (CT) images. Utilizing image registration, the spatial coordinates of the radiosurgical target are compared and relayed to the robotic manipulator, which automatically compensates for off-sets by realigning the linear accelerator's radiation beam. By convention, the total system error of the CyberKnife has been measured using an anthropomorphic head phantom containing a film holder. The x-ray attenuation of the phantom and the radiographic appearance of the phantom in CT and x-ray images seek to emulate the human anatomy.

\section{Materials And Methods}

The Ball-Cube-II film cassette (Figure 1) is a Quality Assurance (QA) tool. Similar to the existing Ball-Cub-I design (Figure 2), the Ball-Cube-II holds two pieces of notched radiochromic film that are placed orthogonally in the film cassette. There are holes and pins in the film Ball-CubeII cassette and holes in the EBT2 film, allowing the film to fit tightly, thus minimizing movement of the film with respect to the film cassette. Determinations of accuracy are similar for both designs; specifically, the spatial coordinates for the centroid of the planned dose distribution is compared with the centroid of the dose actually delivered. In this study, skull tracking mode was used for both Ball Cube measurements.

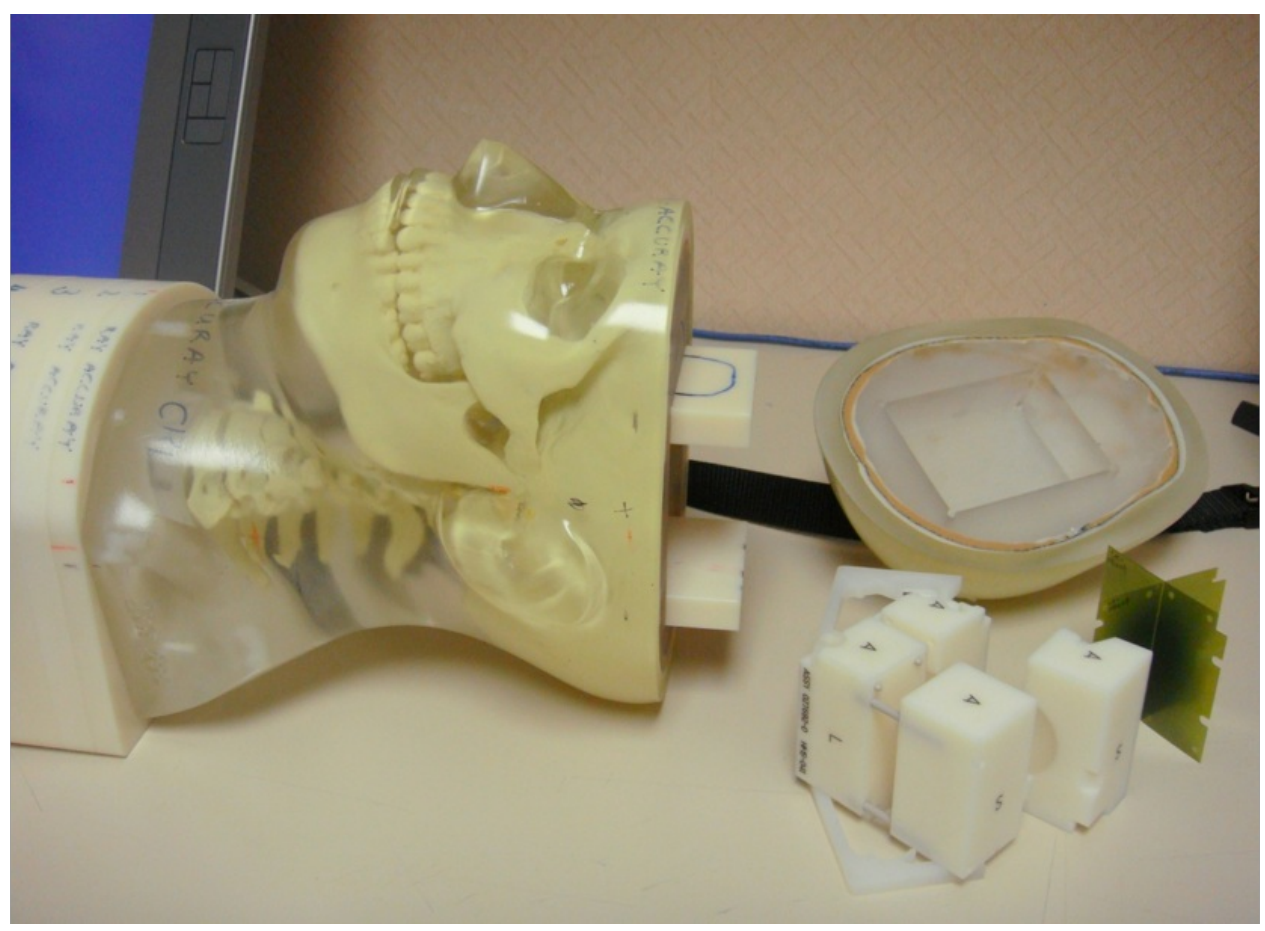

FIGURE 1: Ball-Cube-II film cassette 


\section{Cureus}

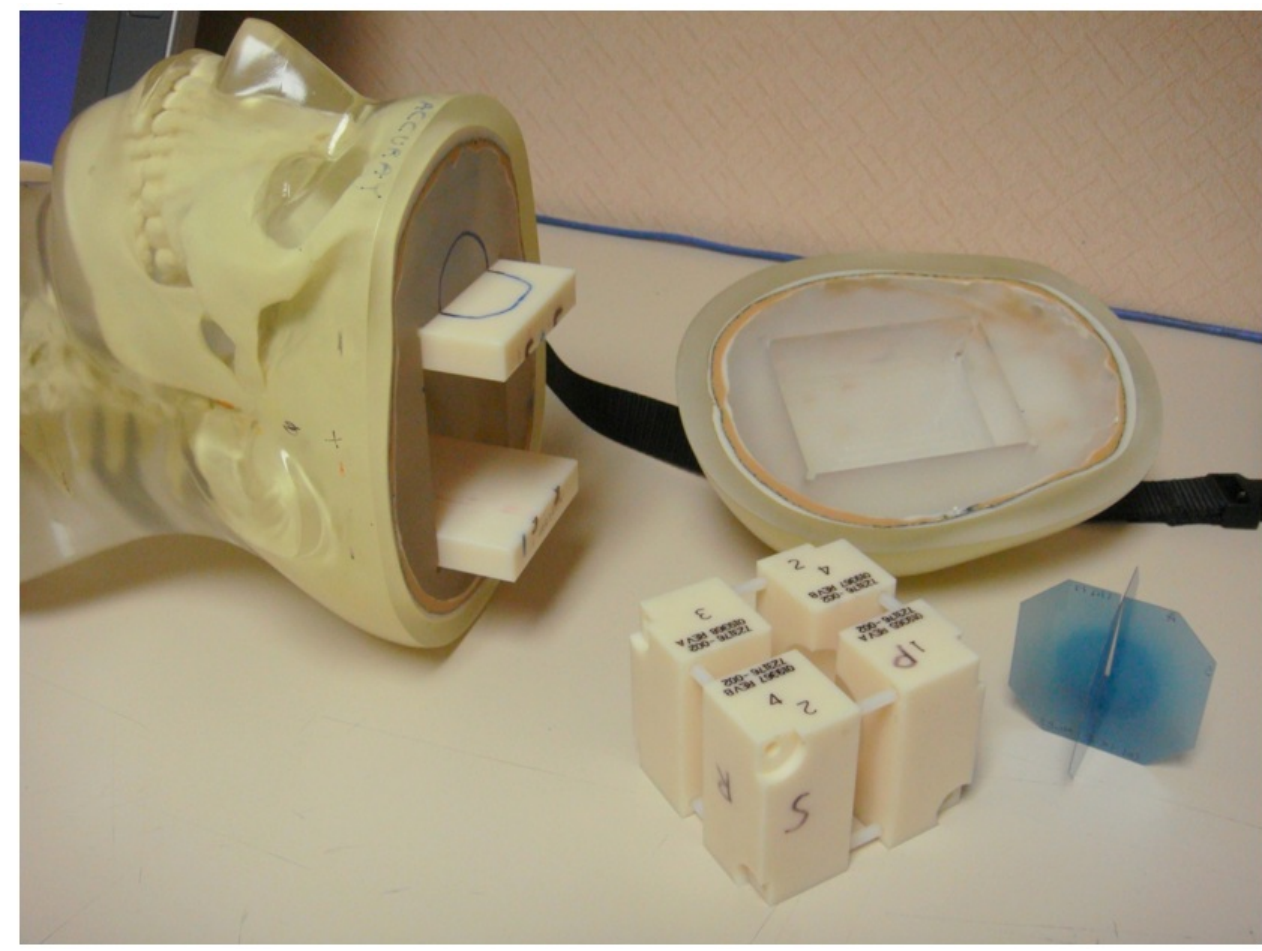

FIGURE 2: Ball-Cube-I film cassette

Accuracy measurements with Ball-Cube-I were performed with MD55 film. Meanwhile, film analysis was done using Accuray E2E software, the output of which establishes total system accuracy. Treatment planning was done with a three path node set, using an isocentric configuration and a $30 \mathrm{~mm}$ collimator. Sixty-two beams were utilized to deliver $2400 \mathrm{cGy}$ to the $70 \%$ isodose line, with a maximum dose of $3000 \mathrm{cGy}$.

The Ball-Cube-II was used with EBT2 film. A treatment plan was constructed with a one path node set, a $30 \mathrm{~mm}$ collimator and conformal planning method. Four hundred and twenty cGy was delivered by means of 71 beams to the $70 \%$ isodose line, with a maximum dose of $600 \mathrm{cGy}$. Within the ball-cube, the two pieces of film are cut in different shapes, one each for the AS (anterior-superior) orientation and AL (anterior-left) orientation. Each piece of film has corresponding laser cut notches and registration holes to facilitate precise and reproducible positioning. Because of the registration holes, the film fits much more tightly onto the alignment pins thereby minimizing movement with respect to the cube. After irradiation was complete, film analysis was done using Accuray's End-to-End (E2E) Film Analysis 4.0 software tool in conjunction with an Epson flat-bed film scanner, model Expression 1680. The EBT2 films were scanned with 48-bit RGB colour mode and analyzed in the red channel.

\section{Results}

\section{Accuracy measurement with Ball-Cube-I and MD-55 film}

A total of 12 measurements were made between October 2008 and June 2009. Data obtained from MD-55 film inserted into the Ball-Cube-I within the head phantom determined the total system inaccuracy to be $0.34+/-0.17 \mathrm{~mm}$. Table 1 shows the results of the accuracy measurement using Ball-Cube-I with the MD-55 films. 


\section{Cureus}

\begin{tabular}{|c|c|c|c|c|c|c|}
\hline & Left & Ant & Sup & Ant & Ave Ant & Total Error \\
\hline Average & 0.20 & -0.01 & -0.13 & -0.10 & -0.06 & 0.34 \\
\hline Std dev & 0.20 & 0.17 & 0.20 & 0.10 & 0.12 & 0.17 \\
\hline
\end{tabular}

TABLE 1: CyberKnife accuracy $(\mathrm{mm})$ measurement with Ball-Cube-I and MD-55 films

\section{Accuracy measurement with Ball-Cube-II and EBT2 film}

Utilizing the EBT2 film inserted into the Ball-Cube II within the head phantom, six accuracy measurements were made from September to October 2009. The total inaccuracy was determined to be $0.45+/-0.09 \mathrm{~mm}$. Table 2 shows these measurements made with the BallCube-II and EBT2 films.

\section{Discussion}

Treatment planning with the Ball-Cube-I and Ball-Cube-II is done differently. Hence, it is not advisable to directly compare the results of the two accuracy measurements reported here. There are uncertainties in contouring the target using the two different systems, while the differing imaging characteristics of the two types of film could also influence accuracy measurements. Nevertheless, the design of the Ball-Cube-II represented a seeming improvement for the placement of film within the film cube. The current study was conducted to see if such a theoretical improvement was beneficial in practice.

Although an experienced person can place the film rather reliably within the Ball- Cube-I, the design of this device introduces greater uncertainty than the Ball-Cube-II. This point is illustrated by the fact that measurements with the Ball-Cube-I yield a standard deviation of $0.17 \mathrm{~mm}$, while the corresponding measure with the Ball-Cube-II is only $0.09 \mathrm{~mm}$. It does not mean that the Ball-Cube-I measurement is inaccurate, just less reproducible, especially for less experienced operators who are not adept at aligning the edges of the films with those of the cube.

The CyberKnife manipulator and imaging subsystems are independently calibrated to a single point, termed the imaging center or machine center. The Deltaman adjustment is a correction that accounts for any systematic translational offset between the two subsystems. This correction is part of the system calibration files that reside on the CyberKnife system treatment delivery system. The results of multiple E2E tests can be used for the Deltaman adjustment. For example, when we check the results of multiple E2E tests for the "Left" error, i.e. $0.2 \mathrm{~mm}$ and $0.34 \mathrm{~mm}$, using Ball-Cube-I and Ball-Cube-II, respectively, we could apply the correction of 0.2 $\mathrm{mm}$ to the Deltaman file. The results of such a process can be used to further augment the targeting accuracy of the CyberKnife system. In doing so, error can be reduced to less than the $0.34 \mathrm{~mm}$ to $0.45 \mathrm{~mm}$ we were able to measure with the two different ball cubes.

\section{Conclusions}

The accuracy of the CyberKnife was determined to be between 0.34 and $0.45 \mathrm{~mm}$. Because the Ball-Cube-II allows minimal movement of the film inside the film cube, it may permit a more precise determination of the CyberKnife's targeting accuracy. 


\section{Cureus}

\section{Additional Information}

\section{Disclosures}

Human subjects: All authors have confirmed that this study did not involve human participants or tissue. Animal subjects: All authors have confirmed that this study did not involve animal subjects or tissue. Conflicts of interest: In compliance with the ICMJE uniform disclosure form, all authors declare the following: Payment/services info: All authors have declared that no financial support was received from any organization for the submitted work. Financial relationships: All authors have declared that they have no financial relationships at present or within the previous three years with any organizations that might have an interest in the submitted work. Other relationships: All authors have declared that there are no other relationships or activities that could appear to have influenced the submitted work.

\section{References}

1. Adler JR Jr., Murphy MJ, Chang SD, Hancock SL: Image-guided robotic radiosurgery. Neurosurgery. 1999, 44:1299-1306.

2. Chang SD, Main W, Martin DP, Gibbs IC, Heilbrun MP: An analysis of the accuracy of the CyberKnife: A robotic frameless stereotactic radiosurgical system. Neurosurgery. 2003, 52:140-146. 